\title{
“拟双子”阴离子表面活性剂在癸烷/水界面的分子动力学模拟
}

\author{
高斯萌* 夏坤康志红 乃永宁袁瑞霞牛瑞霞* \\ (东北石油大学化学化工学院 大庆 163318)
}

\begin{abstract}
摘要 采用全原子分子动力学模拟方法研究了壬基酚取代的系列烷基磺酸盐表面活性剂在癸烷/水界面的微观聚集行 为, 通过分析界面厚度、界面生成能和界面张力以及表面活性剂分子与水分子之间的径向分布函数和配位数，讨论了 不同磺烷基链长度对壬基酚基取代烷基磺酸盐表面活性剂界面性质的影响. 结果表明, 磺烷基链长为 12 时, 表面活性 剂的界面张力最低, 界面厚度和界面生成能最大.
\end{abstract}

关键词 壬基酚取代烷基磺酸盐; 分子动力学; 界面性质; 油水界面; 聚集行为

\section{Molecular Dynamics Simulation of "Quasi-Gemini" Anionic Surfactant at the Decane/Water Interface}

\author{
Gao, Simeng* Xia, Kun Kang, Zhihong Nai, Yongning \\ Yuan, Ruixia Niu, Ruixia* \\ (College of Chemistry and Chemical Engineering, Northeast Petroleum University, Daqing 163318)
}

\begin{abstract}
Anionic surfactants play a key role in many industrial fields such as drug delivery, detergent, oil displacement and food processing because of their unique amphiphilic properties. The structure of surfactant in oil-water system has a great influence on the interfacial behavior. It is of great significance to study the structure and interfacial properties of surfactants. In this paper, the all-atomic molecular dynamics method was used to study the aggregation behavior of nonylphenolsubstituted series of alkyl sulfonate surfactants $\left(\mathrm{C}_{n}\right.$-NPAS) at the decane/water interface. The effects of different sulfoalkyl chain lengths on the interfacial properties of nonylphenol-substituted alkyl sulfonate surfactants were investigated by analyzing the interface thickness, interface formation energy, interfacial tension, the radial distribution function and coordination number. Simulation results have shown that the interfacial thickness increases at first and then decreases as the length of sulfoalkyl chain increases. The same trend was found in the results of the interface formation energy (IFE). The absolute value of IFE follows the order of $\mathrm{C}_{12}$-NPAS $>\mathrm{C}_{14}$-NPAS $>\mathrm{C}_{10}$-NPAS $>\mathrm{C}_{16}$-NPAS $>\mathrm{C}_{8}$-NPAS, indicating that the $\mathrm{C}_{12}$-NPAS is the most stable system in terms of energy which should be attribute to the stronger aggregation ability. Moreover, it is observed that the trend of interfacial tension is in agreement with that of interface formation energy and the interface thickness. Surfactant $\mathrm{C}_{12}$-NPAS induces the minimum interfacial tension. The calculation results are consistent with the experimental data. Furthermore, the radial distribution function and the coordination number of water around the surfactant headgroup were obtained for evaluating the interaction strength between the hydrophilic headgroup and water molecules. The results are well in accordance with the trend of the interface formation energy and interfacial tension. This indicates that the length of alkyl tail affect the interaction between hydrophilic headgroup and water indirectly. Simulation results suggest that the length of alkyl tail plays a dominant role in the interfacial behaviors. We expect that the results of this study could be valuable for the understanding of mechanism and the design of high performance surfactants.

Keywords nonylphenol substituted alkyl sulfonate; molecular dynamics; interface property; oil-water interface; aggregation behavior
\end{abstract}

\section{1 引言}

表面活性剂由于其独特的两亲特性及其在溶液中 的特殊存在方式、物理化学作用, 应用范围日益广泛, 主要用于日化、洗涤、农药、纺织、石油、页岩气等行 业 $^{[1 \sim 6]}$. 在石油工业领域, 表面活性剂几乎应用于各个 生产环节, 已成为石油、页岩气开采中必不可少的化学 品 ${ }^{[7}$ 12]. 理解表面活性剂产生界面性能的基本原理对于
设计新型高效的驱油表面活性剂具有十分重要的作用, 近年来, 许多科学家对表面活性剂的特性进行了广泛的 实验研究.

随着计算机的快速发展, 许多研究者将分子动力学 方法用于研究油/水界面的微观作用机理和界面性质, 利用全原子分子动力学模拟技术，可以在原子水平研究 表面活性剂的双亲结构对其物理化学性质的影响. He课

* E-mail: gaosimeng2006@126.com; niuruixia999@sina.com

Received October 12, 2019; published January 10, 2020.

Project supported by the National Natural Science Foundation of China (No. 21606042), Northeast Petroleum University Cultivation Fund (No. 2017PYQZL-08) and Northeast Petroleum University Talent Engineering Research Start Fund (No. RC201724).

项目受国家自然科学基金(No. 21606042)、东北石油大学培育基金(No. 2017PYQZL-08)和东北石油大学人才工程科研启动基金(No. RC201724)资助. 
题组 ${ }^{[1]}$ 采用分子动力学模拟的方法研究了系列线性烷基 苯磺酸盐在空气/水界面的聚集行为, 结果表明表面张 力与烷基尾部的支化度及尾链长度有关. Jang 课题组 ${ }^{[13]}$ 采用分子动力学模拟方法研究了疏水尾链的异构化对 癸烷/水界面上烷基苯磺酸盐界面性质的影响, 结果表 明中等支化异构体 $4-\mathrm{C}_{16}$ 的有效支链长度与油分子相近, 可能是导致界面处表面活性剂排布更紧密和产生超低 的界面张力的主要原因. 孙课题组 ${ }^{[14]}$ 采用分子动力学 模拟研究了羟基取代的烷基苯磺酸盐单层膜在水/气和 水/癸烷界面的密度分布、氢键结构和表面活性剂聚集结 构的有序度, 探讨了表面活性剂结构和性质之间的关 系. 石课题组 ${ }^{[15]}$ 采用分子动力学的方法研究了阴离子 表面活性剂十六烷基苯磺酸盐在正十六烷一水界面上的 聚集结构, 研究了苯环在碳氢链上的取代位置对油水界 面性质的影响. 王课题组 ${ }^{[16]}$ 采用分子动力学模拟方法 考察了 2-羟基-3,5-烷基苯磺酸钠表面活性剂分子在水/ 气界面形成单层膜的过程, 结果表明两条烷烃链间的相 互作用增强了溶剂对表面活性剂分子的疏水作用.

在前期的工作中, 牛课题组 ${ }^{[17]}$ 合成了一类具有 “拟 双子” 结构的壬基酚取代烷基磺酸盐表面活性剂, 采用 旋转滴法测定了芳基烷基磺酸盐 $\left(\mathrm{C}_{n}\right.$-NPAS, $n=8,10$, $12,14,16)$ 水溶液的油-水动态界面张力, 考察了表面活 性剂烷基链长对油一水动态界面张力的影响, 结果表明 $\mathrm{C}_{12}$-NPAS 体系能够产生最低界面张力. 油水体系中表 面活性剂的结构对界面行为有很大的影响 ${ }^{[18]}$, 研究表 面活性剂结构与界面性质的关系具有重要意义. 本工作 以壬基酚取代的烷基磺酸盐表面活性剂为研究对象, 采 用全原子分子动力学方法模拟了壬基酚取代的烷基磺 酸盐在癸烷/水界面的微观聚集行为, 以期能够从分子 水平上探讨疏水尾链长度对癸烷/水界面行为的影响, 为实验进一步研究提供理论依据.

\section{2 模拟方法}

图 1 为不同碳链长度的壬基酚取代烷基磺酸钠表面 活性剂的构型示意图, 记作 $\mathrm{C}_{n}$-NPAS $(n=8,10,12,14$, 16). 使用 packmol 程序 ${ }^{[19]}$ 构建了油/表面活性剂/水/表面 活性剂/油的双界面模型, 其中包含了 10700 个水分子以 及单侧 700 个正癸烷分子, 油/水两界面各包含 65 个表 面活性剂分子. 为保持体系的电中性, 向水溶液中加入 了 260 个 $\mathrm{Na}^{+}$. 体系总尺寸为 $8.0 \mathrm{~nm} \times 8.0 \mathrm{~nm} \times 15 \mathrm{~nm}$. 所有模型均采用三维周期性结构, 以消除边界效应的影 响.

全部模拟计算使用 gromacs2016 程序 ${ }^{[20]}$ 完成, 所有 原子均采用 GAFF 力场 ${ }^{[21]}$ 进行模拟, 水分子采用 SPCE 模型, 原子电荷使用约束静电势(RESP)方法得到. 对于 每一个体系, 首先采用最速下降法进行能量最小化. 随 后采用分子动力学方法在 $101.325 \mathrm{kPa} 、 298 \mathrm{~K}$ 条件下分 别进行正则系综(NVT)和等温等压系综(NPT)模拟各 10 ns. 压力和温度分别采用 Berendsen 恒压和 Velocityrescale 恒温控制方法, 弛豫时间分别为 1.0 和 $0.1 \mathrm{ps}$. 模 拟中分子的键长使用 LINCS 算法进行约束. LennardJones 相互作用的截止半径为 $1.2 \mathrm{~nm}$. 使用 Particle-Mesh Ewald (PME) 求和法对静电相互作用的长程部分进行了 计算. 最后在 NPT 系综下对各体系进行了 $30 \mathrm{~ns}$ 的分子 动力学模拟, 模拟步长为 $2 \mathrm{fs}$. 通过 $30 \mathrm{~ns}$ 时长的模拟, 体系势能、模拟盒子维度和界面张力等参数保持稳定. 模拟时间最后的 $10 \mathrm{~ns}$ 轨迹用来分析. 采用 VMD 1.9 .1 可视化程序观察动力学轨迹.
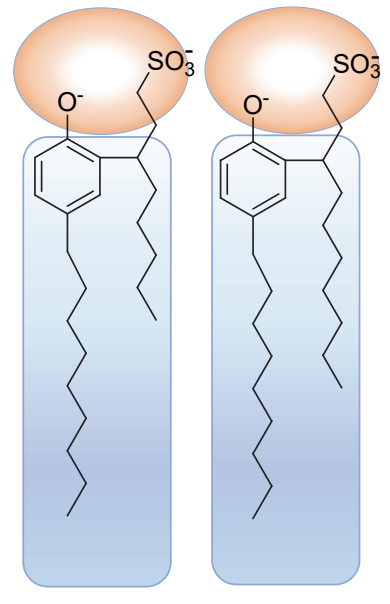

$\mathrm{C}_{8}$-NPAS $\mathrm{C}_{10}$-NPAS
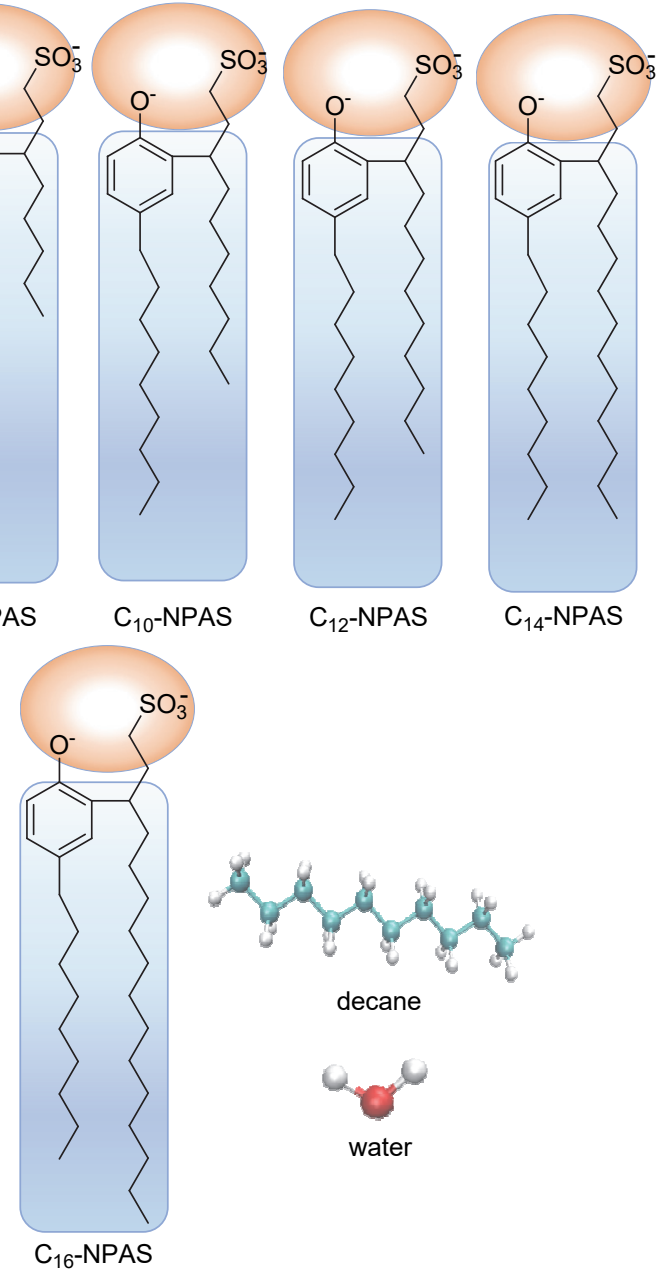

图 1 表面活性剂、正癸烷和水分子的构型图

Figure 1 Molecular structures of surfactants, decane and water molecule

\section{3 结果与讨论}

\section{1 界面形态及密度分布}

以 $\mathrm{C}_{12}$-NPAS 为例讨论表面活性剂单分子层的聚集 结构, 图 2(A)和(B) 分别为 $\mathrm{C}_{12}$-NPAS 体系的初始和平衡 状态的界面聚集结构, 从图中可以看出, 在体系达到平 衡之后呈现出明显的两相界面，表面活性剂分子均聚集 在正癸烷与水的界面处，排布紧密. 两个亲水头基伸向 
水相, 两条疏水烷基尾链伸向油相一侧. 从图 2(A)可以 看出, 在初始结构中, $\mathrm{Na}^{+}$均匀分布在水相, 平衡后由于 表面活性剂极性头基阴离子与 $\mathrm{Na}^{+}$的静电引力的作用, 使得 $\mathrm{Na}^{+}$迁移到界面处, 主要聚集在表面活性剂的极性 头基附近, 有极少量分布在水相. 此外, 从图 2(B)可以 看出, 平衡后还有少量的水分子进入到表面活性剂分子 层中.

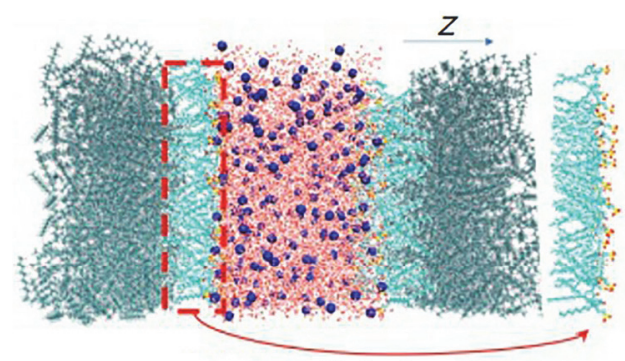

(A) $t=0 \mathrm{~ns}$

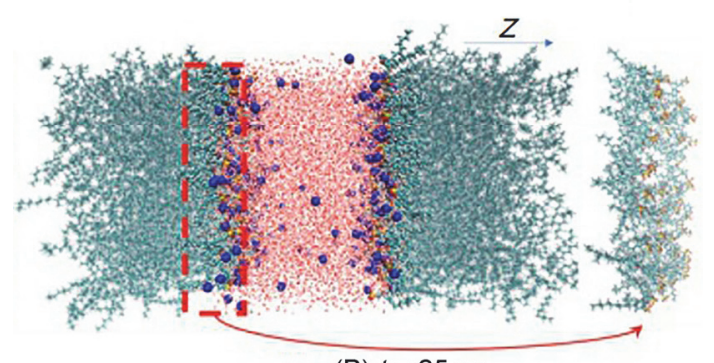

(B) $t=25 \mathrm{~ns}$

图 $2 \mathrm{C}_{12}$-NPAS 体系的初始(A)和平衡(B)构型

Figure 2 Initial (A) and balanced (B) configurations for $\mathrm{C}_{12}$-NPAS system

为了更清晰地分析体系中各组分的分布情况, 图 3 给出了平衡后体系中各组分沿 $Z$ 轴方向的密度分布. 从 图中可以看出, 表面活性剂头基处于水相中, 表明亲水 头基进入到水层发生水化作用. 带正电的 $\mathrm{Na}^{+}$分布在带 负电的头基周围, 说明由于静电相互作用, $\mathrm{Na}^{+}$与表面 活性剂极性头基结合. 经过计算得知, 每个体系中, 油 相平均密度均接近 $718 \mathrm{~kg} / \mathrm{m}^{3}$, 水相的密度接近 1000 $\mathrm{kg} / \mathrm{m}^{3}$, 这与 $298 \mathrm{~K}$ 下正癸烷 $\left(725 \mathrm{~kg} / \mathrm{m}^{3}\right)^{[22]}$ 和水(998 $\left.\mathrm{kg} / \mathrm{m}^{3}\right)^{[23]}$ 的实验密度相符, 说明模拟体系的模型构建合 理, 力场选取和参数设置合适, 动力学模拟结果可以反 映各个体系的界面现象和聚集行为.

\section{2 界面厚度}

体系的界面厚度可以直观地反映出界面吸附的强 弱, 是考察界面性质的一个重要指标, 体系的界面厚度 数值越大, 表明界面活性越强 ${ }^{[13]}$. 对于油/表面活性剂/ 水体系界面，通常采用 “ $90 \% \sim 90 \%$ ” 原则对界面厚度 进行计算 ${ }^{[24]}$, 即采用油相密度的 $90 \%$ 到水相密度 $90 \%$ 之 间的距离定义为界面厚度, 如图 4 所示. 界面厚度包含 三部分, 分别是表面活性剂的厚度 $\sigma_{\text {surfactant, }}$ 水相的厚度 $\sigma_{\mathrm{water}}$ 以及油相的厚度 $\sigma_{\mathrm{oil}}$, 与油/表面活性剂/水的总界面 厚度 $\sigma_{\text {total }}$ 之间的关系可以由公式(1)定义. $\sigma_{\text {surfactant }}=\sigma_{\text {total }}-\sigma_{\text {oil }}-\sigma_{\text {water }}$
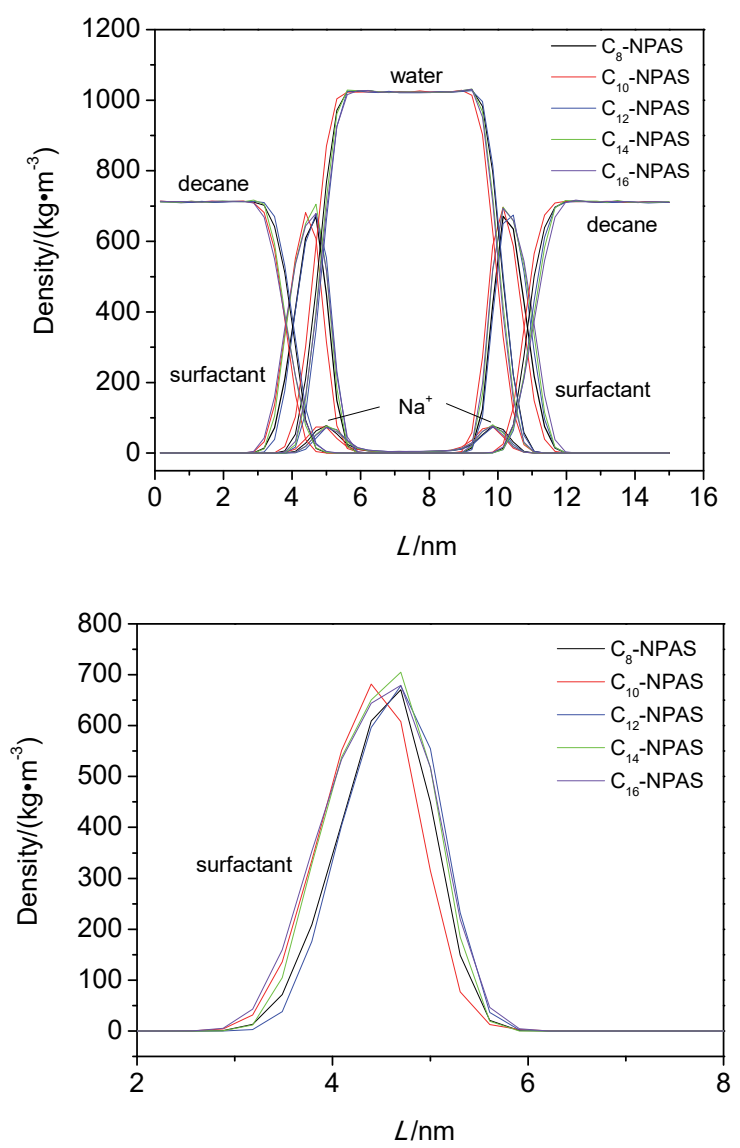

图 $3 \mathrm{C}_{n}$-NPAS 体系不同组分沿 $Z$ 轴方向的密度分布

Figure 3 Density profiles of different system components $Z$ axial direction for $\mathrm{C}_{n}$-NPAS system

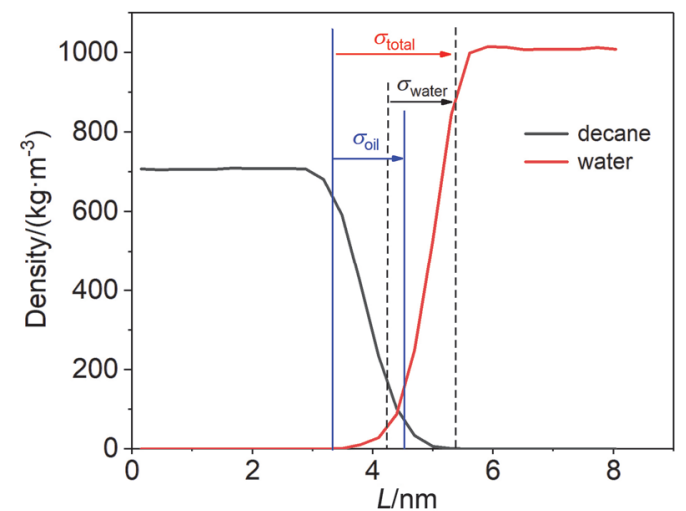

图 4 界面厚度定义

Figure 4 Definition of interfacial thickness

其中油相和水相的厚度分别为油相和水相密度的 10\%到 90\%之间的距离. 依据公式(1)计算得到了不同表 面活性剂在油水界面的总界面厚度，结果如图 5 所示, 没有表面活性剂的情况下，正癸烷/水体系的界面厚度 为 $3.77 \AA$, 加入表面活性剂后, 两个烷基尾链和两个亲 
水基团分别渗透到正癸烷和水分子中, 使得体系的界面 厚度均有不同程度的增加. 5 种表面活性剂体系的界面 厚度大小顺序为 $\mathrm{C}_{12}$-NPAS $>\mathrm{C}_{14}$-NPAS $>\mathrm{C}_{10}$-NPAS $>$ $\mathrm{C}_{16}$-NPAS $>\mathrm{C}_{8}$-NPAS, 其中表面活性剂 $\mathrm{C}_{12}$-NPAS 导致 水相厚度值变宽幅度最大, 使得 $\mathrm{C}_{12}$-NPAS 体系的总界 面厚度达到最大值.

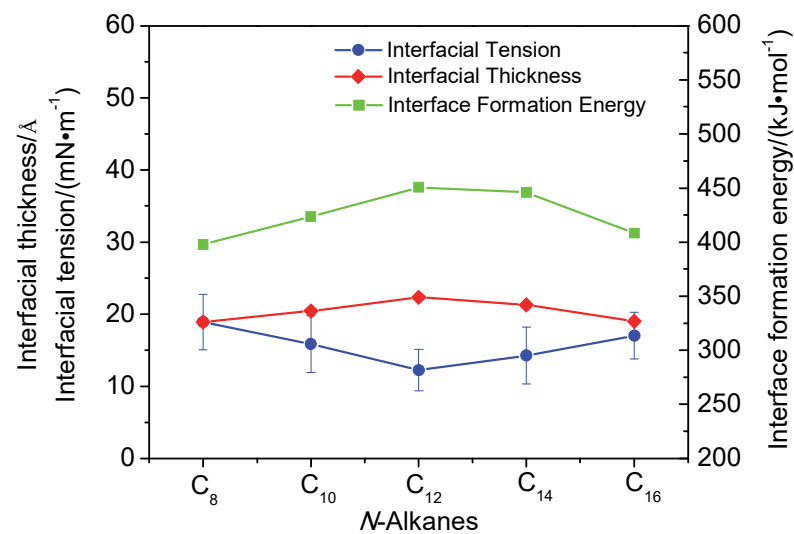

图 $5 \mathrm{C}_{n}$-NPAS $(n=8,10,12,14,16)$ 的界面厚度、界面生成能和界面 张力曲线

Figure 5 Interfacial thickness, interface formation energy and interfacial tension curves of surfactants $\mathrm{C}_{n}$-NPAS $(n=8,10,12,14,16)$

\section{3 界面生成能}

界面生成能能够反映出界面体系的稳定性 ${ }^{[25]}$, 为 比较不同碳链长度对体系界面稳定性的影响, 对各个体 系的界面生成能进行了计算. 界面生成能是指向油水界 面插入 1 个表面活性剂分子后使体系降低的能量, 其值 与表面活性剂极性头基和水分子、疏水尾链和正癸烷分 子以及表面活性剂分子之间的相互作用相关. 计算公式 如下:

$$
I F E=\frac{E_{\text {total }}-E_{\text {decane }- \text { water }}-E_{\text {surfactant }}}{n}
$$

公式(2)中, $E_{\text {total }}, E_{\text {decane- }}{ }^{- \text {water }}$ 和 $E_{\text {surfactant }}$ 分别对应体系 的总能量, 纯正癸烷/水体系的能量以及单个表面活性 剂分子和两个 $\mathrm{Na}^{+}$离子在真空状态下运行 $200 \mathrm{ps}$ 时的能 量. $n$ 为表面活性剂的总数量. 表面活性剂的加入使体 系的总能量降低, 导致界面生成能是一个负值, 其绝对 值越大, 形成的界面就越稳定. 计算得到的界面生成能 如图 5 所示, 界面生成能数值的绝对值趋势为 $\mathrm{C}_{12}-\mathrm{NPAS}$ $>\mathrm{C}_{14}$-NPAS $>\mathrm{C}_{10}$-NPAS $>\mathrm{C}_{16}$-NPAS $>\mathrm{C}_{8}$-NPAS, 其中 $\mathrm{C}_{12}$-NPAS 体系界面生成能的绝对值最大, 表明其界面 最稳定, 表面活性剂分子之间排列最紧密, 最有利于形 成界面，该顺序与界面厚度值顺序相一致.

\section{4 界面张力}

界面张力是三次采油中衡量表面活性剂性能的一 个重要指标 ${ }^{[26]}$, 依据公式(3) ${ }^{[27]}$ 对各体系的界面张力值 进行了计算.

$$
\gamma=\frac{1}{2}\left(p_{z z}-\frac{p_{x x}+p_{y y}}{2}\right) L_{z}
$$

公式(3)中, $p_{z z}$ 是指垂直于界面方向的分压, $p_{x x}$ 和 $p_{y y}$ 是平行于界面方向的分压. $L_{z}$ 是 $z$ 方向的长度. 在没有表 面活性剂时，对纯油/水体系模拟得到的界面张力值为 $(54.23 \pm 2.12) \mathrm{mN} / \mathrm{m}$, 与该温度下的实验值 51.72 $\mathrm{mN} / \mathrm{m}^{[28]}$ 相一致, 说明当前模型和所使用的力场可以较 准确地描述该界面体系. 计算得到的各体系界面张力值 如图 5 所示, 从小到大的排列顺序为 $\mathrm{C}_{12}$-NPAS $<$ $\mathrm{C}_{14}$-NPAS $<\mathrm{C}_{10}$-NPAS $<\mathrm{C}_{16}$-NPAS $<\mathrm{C}_{8}$-NPAS, 该变化 趋势与界面生成能及界面厚度的变化相一致. 其中 $\mathrm{C}_{12}$-NPAS 体系界面张力值最低, 说明 $\mathrm{C}_{12}$-NPAS 表面活 性剂的两条疏水尾链与油相具有良好的相溶性, 反映出 链长对界面性质具有较大影响. 该结果与牛课题组 ${ }^{[17]}$ 的实验研究结论一致, 进一步说明研究体系所使用的模 型和方法能够准确反映出体系的界面性质.

\section{5 径向分布函数}

径向分布函数 $g(r)$ 是描述分子体系结构的一个重要 函数, 可以用来分析液体的微观结构, 通常将其定义为 与 $\mathrm{A}$ 类中心原子距离为 $r$ 的球壳中出现 $\mathrm{B}$ 类原子的概率, 该函数可以反映出两原子之间的相互作用关系 ${ }^{[29]}$. 峰 值的位置对应 A-B 间的最可能距离. 为了获得表面活性 剂亲水头基与水分子之间的相互作用信息, 我们对五个 体系中, 表面活性剂磺酸基中的氧原子 $\left(\mathrm{O}_{\mathrm{S}}\right)$ 和酚羟基中 的氧原子 $\left(\mathrm{O}_{\mathrm{o}}{ }^{-}\right)$分别与水分子中的氧 $\left(\mathrm{O}_{\mathrm{W}}\right)$ 之间的径向分 布函数进行了统计，结果如图 6 所示.

从图 6(A)中看出, 不同碳链长度的表面活性剂体系 的 RDF 很相似, 即第一个峰值均出现在 $(0.236 \pm 0.002)$ $\mathrm{nm}$ 的位置, 同时在 $(0.482 \pm 0.006) \mathrm{nm}$ 处也有一较强峰 值, 两峰之间的距离约为 $0.24 \mathrm{~nm}$, 约等于一个氢键的 距离 $(0.27 \mathrm{~nm})$, 说明第一水化层和第二水化层是通过氢 键的相互作用而结合. 在图 6(B) 中, 具有相似的趋势, 两个峰值分别位于 $(0.232 \pm 0.004) \mathrm{nm}$ 和 $(0.466 \pm 0.021)$ $\mathrm{nm}$ 处, 相似的趋势与刘课题组 ${ }^{[30]}$ 报道相符. 从两图中 第二个峰值出现的高度可以看出, $\mathrm{C}_{12}$-NPAS 体系中两个 亲水基团与水分子的作用最强，可能是导致其形成最低 界面张力的原因.

\section{6 配位数}

为了定量地描述表面活性剂亲水头基与水分子之 间的相互作用，我们采用公式(4)对表面活性剂磺酸基 中的氧和酚羟基中的氧与水分子之间的配位数进行了 计算 ${ }^{[31]}$.

$$
n=\int_{R_{1}}^{R_{2}} 4 \pi r^{2} \rho g(r) \mathrm{d} r
$$



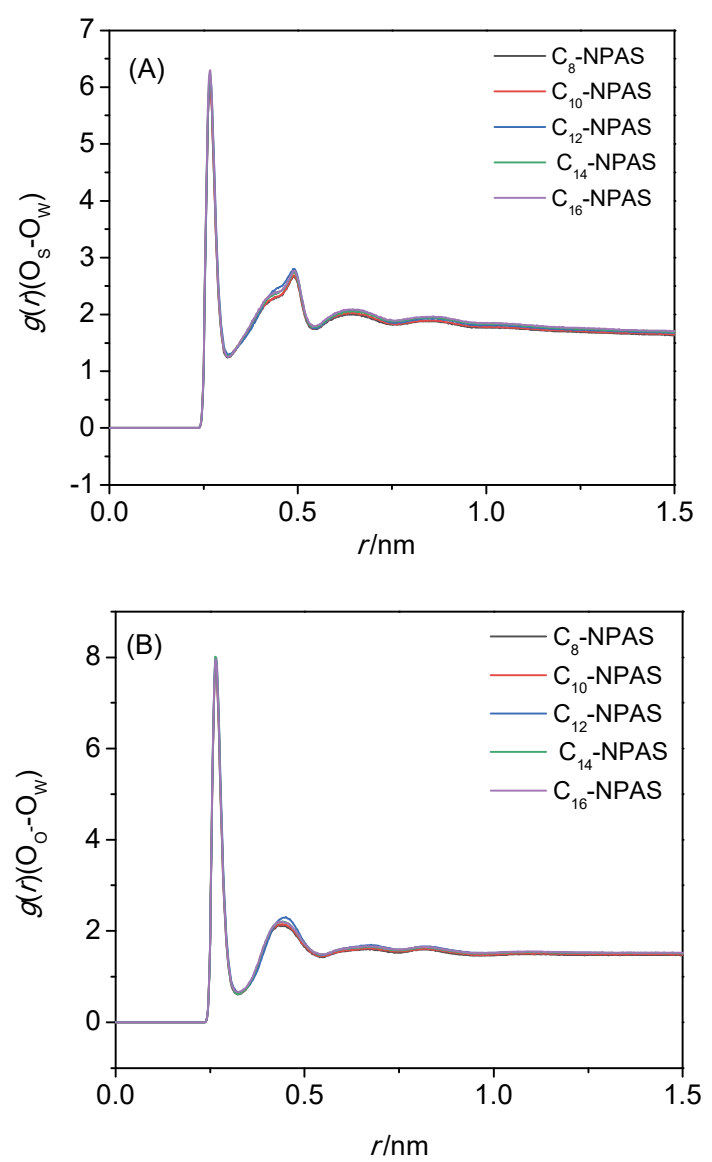

图 6 (A) $\mathrm{O}_{\mathrm{S}}$ 与 $\mathrm{O}_{\mathrm{W}}$ 的径向分布函数; (B) $\mathrm{O}_{\mathrm{O}}{ }^{-}$与 $\mathrm{O}_{\mathrm{W}}$ 的径向分布函数 Figure 6 Radial distribution function. (A) $g(r)\left(\mathrm{O}_{\mathrm{S}}-\mathrm{O}_{\mathrm{W}}\right)$; (B) $g(r)$ $\left(\mathrm{O}_{\mathrm{O}}{ }^{-}-\mathrm{O}_{\mathrm{w}}\right)$

在公式(4)中, $r$ 表示水中氧原子 $\left(\mathrm{O}_{\mathrm{w}}\right)$ 到表面活性剂 磺酸基中的氧 $\left(\mathrm{O}_{\mathrm{S}}\right)$ 和酚羟基中的氧 $\left(\mathrm{O}_{\mathrm{O}}{ }^{-}\right)$的距离. $r$ 为水 相中氧原子的数密度. $g(r)$ 为氧原子与表面活性剂头基 的径向分布函数, $R$ 代表峰的始末. $n$ 表示表面活性剂亲 水头基周围水分子的配位数. 计算得到配位数的数值列 于表 1 中.

表 1 表面活性剂头基周围水分子配位数

Table 1 Coordination number of water molecules around surfactant head group

\begin{tabular}{ccccccc}
\hline & \multicolumn{2}{c}{ First hydration shell } & & \multicolumn{2}{c}{ Second hydration shell } & \multirow{2}{*}{ Total } \\
\cline { 2 - 3 } & $\mathrm{O}_{\mathrm{s}}-\mathrm{O}_{\mathrm{W}}$ & $\mathrm{O}_{\mathrm{O}^{-}-\mathrm{O}_{\mathrm{W}}}$ & & $\mathrm{O}_{\mathrm{s}}-\mathrm{O}_{\mathrm{W}}$ & $\mathrm{O}_{\mathrm{O}^{-}-\mathrm{O}_{\mathrm{W}}}$ & \\
\hline $\mathrm{C}_{8}$-NPAS & 0.21 & 0.25 & & 0.46 & 0.32 & 1.24 \\
$\mathrm{C}_{10}$-NPAS & 0.21 & 0.25 & & 0.45 & 0.36 & 1.27 \\
$\mathrm{C}_{12}$-NPAS & 0.22 & 0.26 & & 0.49 & 0.37 & 1.34 \\
$\mathrm{C}_{14}$-NPAS & 0.22 & 0.25 & & 0.47 & 0.35 & 1.29 \\
$\mathrm{C}_{16}$-NPAS & 0.22 & 0.25 & & 0.46 & 0.34 & 1.27 \\
\hline
\end{tabular}

可以看出随着烷基链长的变化, 尽管每个表面活性 剂亲水基团周围水分子的总配位数相差不大，但是仍然 具有与界面张力、界面生成能和界面厚度相一致的变化 趋势, 说明疏水尾链长度的变化能够影响到亲水头基与 水的作用强度. 其中 $\mathrm{C}_{12}$-NPAS 的亲水基团周围水分子
的配位数最大，说明极性头基周围水分子数最多. 同时 该体系的界面厚度和界面生成能均表现出最大值, 且具 有最低的界面张力. 这是由于 $\mathrm{C}_{12}$-NPAS 体系中表面活 性剂分子的磺酸基尾链由 12 个碳原子构成, 靠近磺酸 基的第三个碳原子与苯环相连接, 其裸露在油相中的有 效碳链包含 10 个碳原子, 与油分子的碳链长度最为相 近，该表面活性剂结构中的疏水尾链与油分子之间具有 最好的相溶性, 与油分子之间相互作用力最强, 因此界 面生成能最大, 界面张力最低, 表面活性剂在界面聚集 得最为紧密. 分子之间作用力的增强, 增加了分子间极 性头基之间的相互作用，使表面活性剂分子中两个亲水 头基之间形成了相对较大的空间，能够容纳更多的水分 子, 因此该结构亲水头基与水分子的作用力最强, 形成 的径向分布函数峰值最高, 配位数最大.

为了直观地描述水分子在亲水性头基周围的分布 情况，在模拟体系中选取了一个表面活性剂分子和周围 结合的水分子绘制出如图 7 所示的结构图.

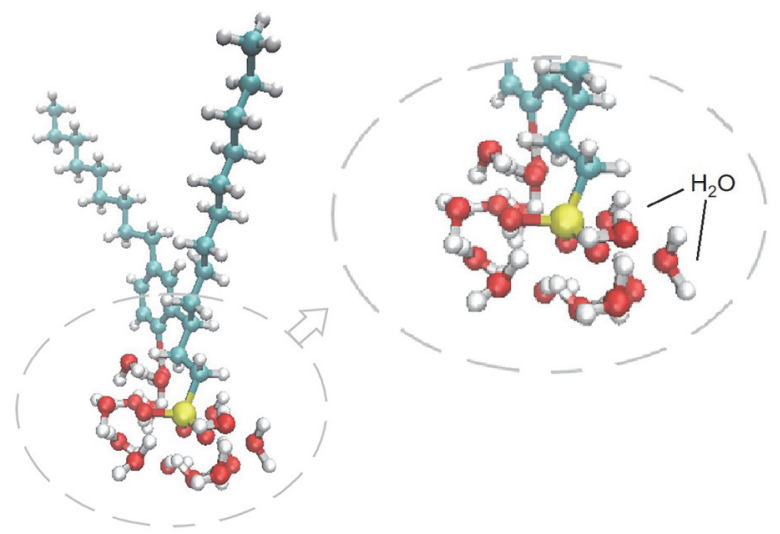

图 7 亲水性头基周围水分子排布

Figure 7 Arrangement of water molecules around hydrophilic head groups

\section{4 结论}

采用全原子分子动力学方法，对壬基酚取代的不同 链长烷基磺酸盐在癸烷/水界面的界面性质进行了模拟. 结果表明，当烷基链长为 12 时，体系具有最大的界面厚 度和界面生成能，以及最低的界面张力，同时与水分子 的结合作用最强, 研究结果与实验研究结果一致, 并对 实验结果从微观层次进行了分析. 本研究工作表明分子 动力学模拟是研究表面活性剂在油/水界面吸附行为的 有力工具, 可用于研究油/水界面的微观作用机理, 并为 三次采油中驱油用表面活性剂的选择和应用提供理论 指导.

\section{References}

[1] He, X. B.; Guvench, O.; Mackerell Jr., A. D.; Klein, M. L. J. Phys. Chem. B 2010, 114, 9787.

[2] Yu, Y. X.; Zhao, J.; Guvench, O.; Bayly, A. E. Chin. J. Chem. Eng. 2008, 16, 517.

[3] Zhao, W. W.; Wang, Y. L. Acta Chim. Sinica 2019, 77, 717 (in Chi- 
nese). (赵微微, 王毅琳, 化学学报, 2019, 77, 717.)

[4] Yan, H.; Guo, X. L.; Liu, C. B.; Yuan, S. L. Langmuir 2011, 27, 5762.

[5] Lin, C.; Pan, R. M.; Xing, P. Chin. J. Org. Chem. 2018, 38, 3260 (in Chinese). (林超, 潘仁明, 邢萍, 有机化学, 2018, 38, 3260.)

[6] Zhao, Y.; Wang, C.; Chow, A. H.; Ren, K.; Gong, T.; Zhang, Z. R.; Zheng, Y. Int. J. Pharm. B 2010, 383, 170.

[7] Liao, G. Z.; Wang, Q.; Wang, H. Z.; Liu, W. D.; Wang, Z. M. Acta Petrolei Sinica 2017, 38, 196 (in Chinese). (廖广志, 王强, 王红庄, 刘卫东, 王正茂, 石油学报, 2017, 38, 196.)

[8] Yu, T.; Liu, H. J.; Ding, W. Chin. J. Appl. Chem. 2008, 25, 1107 (in Chinese). (于涛, 刘红娟, 丁伟，应用化学, 2008, 25, 1107.)

[9] Chen, X. M.; Chen, Y.; Liu, Y. Chin. J. Chem. 2018, 36, 526.

[10] Li, H. F.; Qiao, F. L.; Fan, Y. X.; Wang, Y. L. Acta Chim. Sinica 2018, 76, 564 (in Chinese). (李浩飞, 乔富林, 范雅珣, 王毅琳，化 学学报, 2018, 76, 564.)

[11] Li, M.; Zhang, C.; Yang, X. Chin. J. Chem. 2017, 35, 1706.

[12] Li, Z. Q.; Guo, X. L.; Wang, H. Y.; Li, Q. H.; Yuan, S. L. Acta Phys.-Chim. Sin. 2009, 25, 6 (in Chinese). (李振泉, 郭新利, 王红 艳, 李青华, 苑世领, 物理化学学报, 2009, 25, 6.)

[13] Jang, S. S.; Lin, S. T.; Maiti, P. K.; Blanco, M.; Goddard, W. A.; Shuler, P. J. Phys. Chem. B 2004, 108, 12130.

[14] Sun, H. Q.; Xiao, H. Y.; Liu, X. H. Sci. Chin. Chem. 2011, 54, 1078 (in Chinese). (孙唤泉, 肖红艳, 刘新厚, 中国科学: 化学, 2011, 54,1078 .)

[15] Shi, J.; Lv, K.; Yuan, S. L. J. Shandong Univ. (Engineering Science) 2012, 42, 77 (in Chinese). (石静, 吕凯, 苑世领, 山东大学学报: 工学版, 2012, 42, 77.)

[16] Wang, Y. F.; Yu, W. Z.; Hu, S. Q. J. Chin. Univ. Petro. 2011, 35, 153 (in Chinese). (王业飞, 于维钢, 胡松青, 中国石油大学学报, 2011, 35, 153.)

[17] Niu, R. X.; Wang, D. Q.; Wang, J. L.; Wang, C.; Song, H. J. Chem.
Ind. Eng. 2016, 67, 2944 (in Chinese). (牛瑞霞，王大强，王敬玲， 王超, 宋华, 化工学报, 2016, 67, 2944.)

[18] Tan, J. S.; Zhang, L.; Lim, F. C.; Cheong, D. W. Langmuir 2017, 33, 4461.

[19] Martínez, L.; Andrade, R.; Birgin, E. G. J. Comput. Chem. 2009, 30, 2157.

[20] Lundborg, M.; Lindahl, E. J. Phys. Chem. B 2014, 119, 810.

[21] Wang, J.; Wolf, R. M.; Caldwell, J. W.; Kollman, P. A.; Case, D. A. J. Comp. Chem. 2004, 25, 1157.

[22] Sastry, N. V.; Valand, M. K. J. Chem. Thermodyn. 1998, 30, 929.

[23] Pai, Y. H.; Chen, L. J. J. Chem. Eng. Data 1998, 43, 665.

[24] Hou, M. D.; Li, H. P.; Hu, Z. Z.; Song, H. W. China Surfactant Detergent \& Cosmetics 2018, 48, 243 (in Chinese). (候孟蝶, 李惠萍, 胡子昭, 宋宏伟, 日用化学工业, 2018, 48, 243.)

[25] Hu, X. Y.; Song, X. W.; Li, Q. W.; He, X. J.; Li, Y. Acta Chim. Sinica 2009, 67, 1691 (in Chinese). (胡晓莹, 宋新旺, 李全伟, 何秀 娟, 李英, 化学学报, 2009, 67, 1691.)

[26] Olayiwola, S. O.; Dejam, M. Fuel 2019, 241, 1045.

[27] Jiang, R. J.; Luo, J. H.; Bai, R. B.; Jiang, B.; Zhou, G. Chem. J. Chin. Univ. 2017, 38, 1804 (in Chinese). (江蓉君, 罗健辉, 白瑞兵, 江波, 周歌, 高等学校化学学报, 2017, 38, 1804.)

[28] Zeppieri, S.; Rodriguez, J.; López, R. A. J. Chem. Eng. Data 2001, 46, 1086.

[29] Wang, J.; Wang, J. X.; Zeng, F. G.; Wu, X. L. Acta Chim. Sinica 2010, 68, 1653 (in Chinese). (王进, 王军霞, 曾凡桂, 吴秀玲, 化 学学报, 2010, 68, 1653.)

[30] Liu, M. T.; Pu, M. F.; Ma, H. W. Chem. J. Chin. Univ. 2012, 33, 1319 (in Chinese). (刘梅堂, 浦敏锋, 马鸿文, 高等学校化学学报, 2012, 33, 1319.)

[31] Liu, Z. Y.; Liao, Q.; Jin, Z. Q.; Zhang, L.; Zhang, L. Acta Phys.-Chim. Sinica 2016, 32, 1168 (in Chinese). (刘子瑜，廖琦，靳 志强, 张否, 张路, 物理化学学报, 2016, 32, 1168.)

(Cheng, B.; Fan, Y.) 